\title{
Editorial
}

\section{Networked Dynamical Systems: Analysis and Synthesis}

\author{
Jinde Cao, ${ }^{1,2}$ Guoqiang Hu, ${ }^{3}$ Wei Lin, ${ }^{4}$ Guanghui Wen, ${ }^{1}$ \\ Wenwu Yu, ${ }^{1,5}$ and Zhiqiang $\mathrm{Zuo}^{6}$ \\ ${ }^{1}$ Department of Mathematics, Research Center for Complex Systems and Network Sciences, Southeast University, \\ Nanjing 210096, China \\ ${ }^{2}$ Department of Mathematics, Faculty of Science, King Abdulaziz University, Jeddah 21589, Saudi Arabia \\ ${ }^{3}$ School of Electrical and Electronics Engineering, Nanyang Technological University, Singapore 639798 \\ ${ }^{4}$ School of Mathematical Sciences, Fudan University, Shanghai 200433, China \\ ${ }^{5}$ School of Electrical and Computer Engineering, RMIT University, Melbourne, VIC 3001, Australia \\ ${ }^{6}$ School of Electrical Engineering and Automation, Tianjin University, Tianjin 300072, China \\ Correspondence should be addressed to Jinde Cao; jdcao@seu.edu.cn
}

Received 9 June 2014; Accepted 9 June 2014; Published 17 June 2014

Copyright (C) 2014 Jinde Cao et al. This is an open access article distributed under the Creative Commons Attribution License, which permits unrestricted use, distribution, and reproduction in any medium, provided the original work is properly cited.

It is well known that most of the real-world large-scale systems can be modeled as networked dynamical systems where the nodes indicate the individuals while the links mimic the interaction among them. The examples in point are the protein interaction networks, distributed satellite systems, and large-scale sensor systems. In the past few years, collective behaviors of networked dynamical systems have become the subject of hot research in control theory and engineering.

The present special issue focuses on analysis and synthesis of networked dynamical systems. It tries to not only understand the evolution mechanisms responsible for various collective behaviors, but also design some interaction rules such that the desirable collective behaviors can be emerged. Call for papers has been carefully prepared by the guest editors and posted on the journal's web page, which has received much attention from researchers in different scientific communities. We have received 37 papers in this research field. All manuscripts submitted to this special issue went through a thorough peer-refereeing process. Based on the reviewers' reports, 25 original research articles are finally accepted. The contents of this special issue contain modeling and control of complex networks, collective behaviors in multiagent systems, and analysis and synthesis of complex dynamical systems, which are summarized as follows.
(1) Modeling and Control of Complex Networks. "Noise and synchronization analysis of the cold-receptor neuronal network model" by Y. Du et al. studies the synchronization behavior of a class of neuronal networks. Some interesting synchronization conditions are provided. "The Kirchhoff index of hypercubes and related complex networks" by J. Liu et al. gives an exact formula for the Kirchhoff index of the hypercubes networks. In "Modified projective outer synchronization between two fractional order complex networks via adaptive control" by H.-J. Liu et al., the modified projective outer synchronization of a class of fractional order complex networks is given and analyzed. "Moving target detection and active tracking with a multicamera network" by L. Zhao et al. introduces a systematic framework for intelligence video surveillance system (IVSS) with a multicameras network. Specifically, the proposed framework consists of lowcost static and PTZ cameras, target detection and tracking algorithms, and a low-cost PTZ camera feed-back control algorithm based on target information. "Nobelist TD LEE scientist cooperation network and scientist innovation ability model" by J.-Q. Fang and Q. Liu gives some interesting results on nobelist TD Lee scientist cooperation network (TDLSCN). It has been shown in the above-mentioned paper that not only the TDLSCN has the common topological properties of both scale-free property and small-world effect for general 
scientist cooperation networks, but also has the creation of multiple-peak phenomenon for number of published papers with year evolution.

(2) Collective Behaviors in Multiagent Systems. "Consensus analysis for high-order multi-agent systems without or with delays" by Z. Wang and Y. Cao investigates the consensus problem of a class of high-order integrator-type multiagent systems. A necessary and sufficient condition for guaranteeing consensus is established for multiagent systems without delays. Then, some sufficient conditions for consensus of multiagent systems with delays are given. "Opinion impact models and opinion consensus methods in ad hoc tactical social networks" by $\mathrm{D}$. Li et al. analyzes the opinion consensus in ad hoc tactical social networks.

(3) Analysis and Synthesis of Complex Dynamical Systems. "Finite-time stability and stabilization of networked control systems with bounded Markovian packet dropout" by Y. Sun and G. Li studies the finite-time stability and stabilization problem of networked linear systems in the presence of Markovian packet dropout. "Adaptive fractional fuzzy sliding mode control for multivariable nonlinear systems" by J. Luo and $\mathrm{H}$. Liu investigates the stabilization problem of a class of nonlinear systems.

Note that the selected topics and papers are not a comprehensive representation of the area of the special issue. It is also worth noting that the published papers in this special issue do provide some recent advances in the field of networked dynamical systems, which could benefit the current research more or less.

\section{Acknowledgments}

We would like to thank the authors for their contributions. We also thank the anonymous reviewers for their time and help in assessing the manuscripts. Our special thanks are due to the editorial board members of this journal owing to their great support and help for this special issue.

Jinde Cao

Guoqiang $\mathrm{Hu}$

Wei Lin

Guanghui Wen

Wenwu Yu

Zhiqiang Zuo 


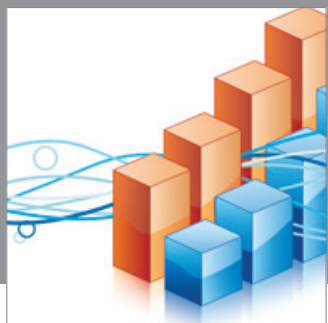

Advances in

Operations Research

mansans

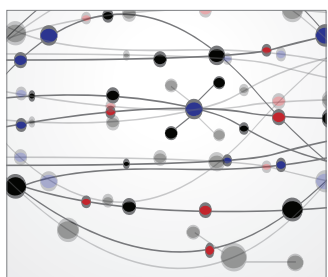

The Scientific World Journal
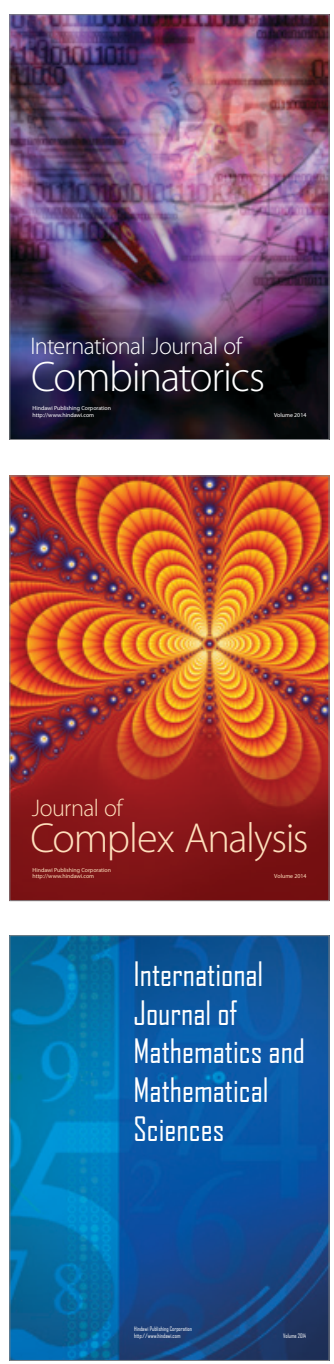
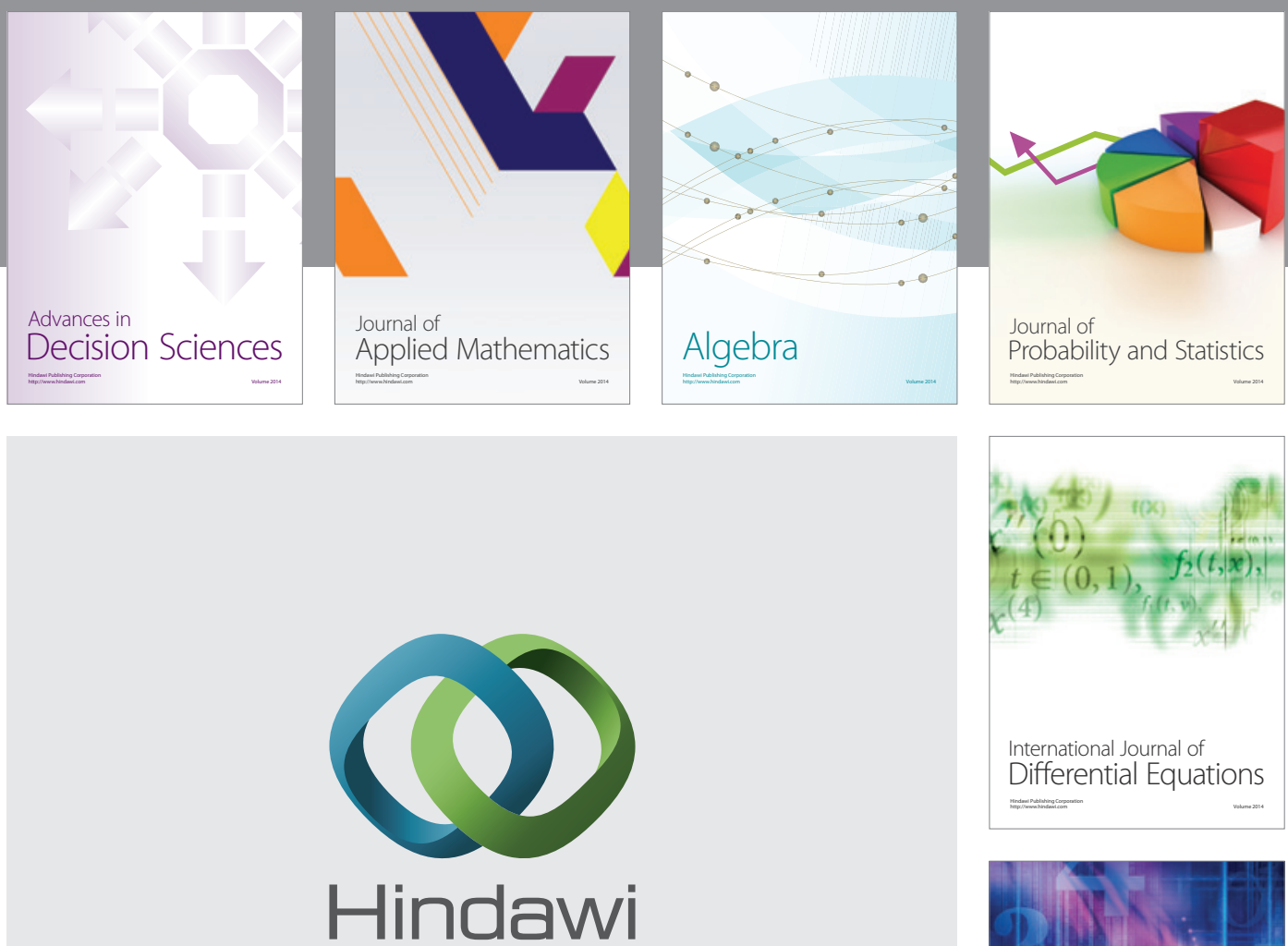

Submit your manuscripts at http://www.hindawi.com
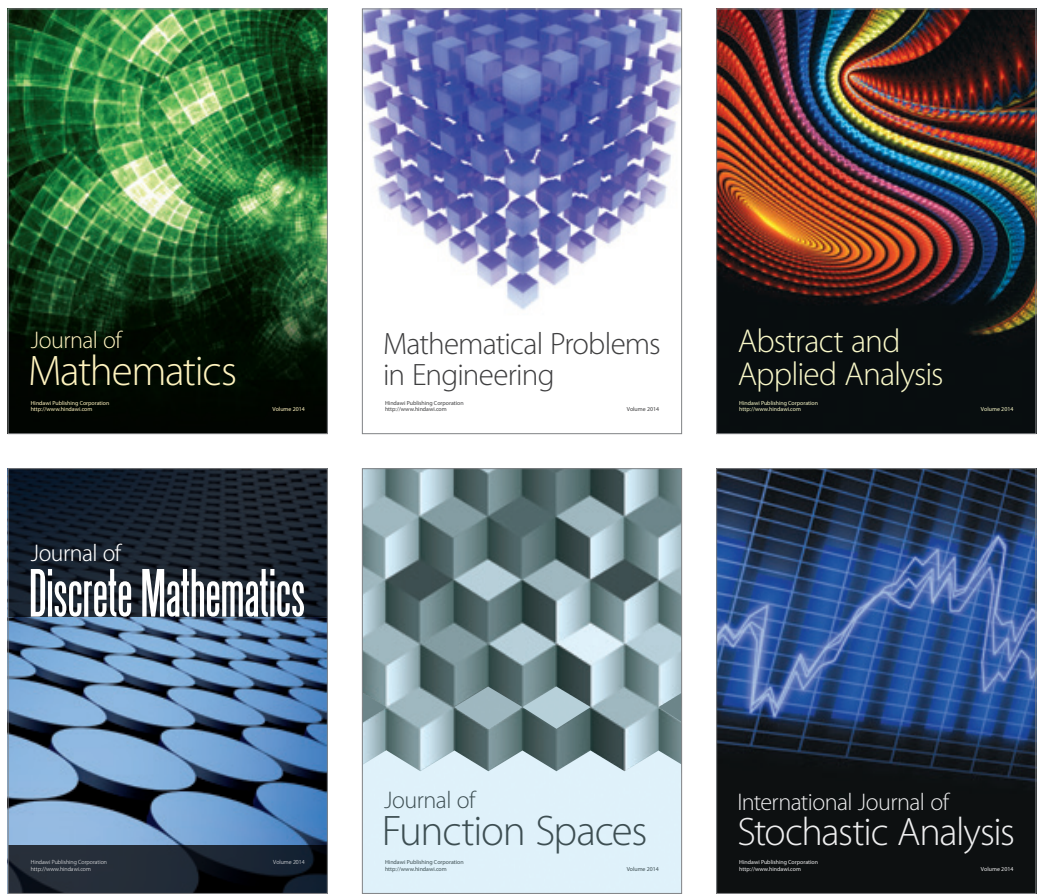

Journal of

Function Spaces

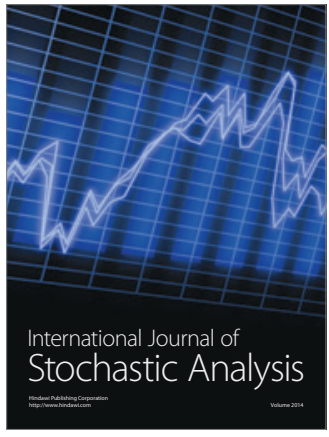

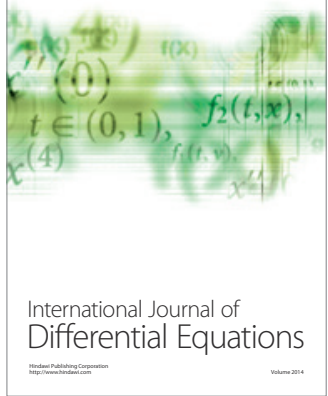
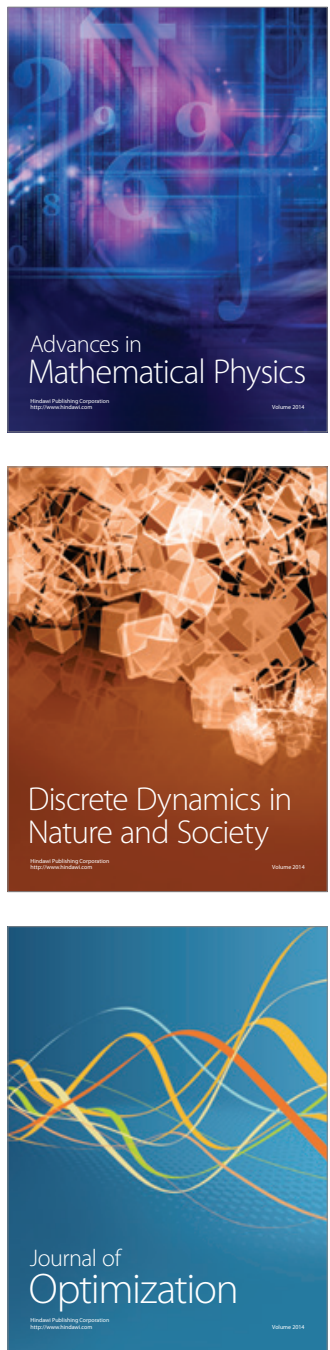